OPEN ACCESS

Edited by:

Marie-France Penet,

Johns Hopkins University,

United States

Reviewed by:

Andrea Papadia,

University of Italian Switzerland,

Switzerland

Boudewijn Schaafsma, Albert Schweitzer Ziekenhuis, Netherlands

*Correspondence: Alexander L. Vahrmeijer A.L.Vahrmeijer@/umc.nl

Specialty section:

This article was submitted to Cancer Imaging and Image-directed Interventions,

a section of the journal

Frontiers in Oncology

Received: 22 July 2020 Accepted: 09 November 2020 Published: 17 December 2020

Citation:

Baart VM, Deken MM, Bordo MW, Bhairosingh SS, Salvatori DCF,

Hyun H, Henary M, Choi HS, Sier CFM, Kuppen PJK, van Scheltinga AGTT, March TL, Valentijn ARPM, Frangioni $J$ and Vahrmeijer AL (2020) Small Molecules for Multi-Wavelength Near-Infrared

Fluorescent Mapping of Regiona and Sentinel Lymph Nodes in

Colorectal Cancer Staging.

Front. Oncol. 10:586112.

doi: 10.3389/fonc. 2020.586112

\section{Small Molecules for Multi- Wavelength Near-Infrared Fluorescent Mapping of Regional and Sentinel Lymph Nodes in Colorectal Cancer Staging}

\author{
Victor M. Baart ${ }^{1}$, Marion M. Deken ${ }^{1}$, Mark W. Bordo ${ }^{2}$, Shadhvi S. Bhairosingh ${ }^{1}$, \\ Daniela C. F. Salvatori ${ }^{3,4}$, Hoon Hyun ${ }^{5}$, Maged Henary ${ }^{6}$, Hak Soo Choi ${ }^{7}$, \\ Cornelis F. M. Sier ${ }^{1}$, Peter J. K. Kuppen ${ }^{1}$, Anton G. T. Terwisscha van Scheltinga ${ }^{8}$, \\ Taryn L. March ${ }^{8}$, Adrianus R. P. M. Valentijn ${ }^{8}$, John V. Frangioni ${ }^{2}$ \\ and Alexander L. Vahrmeijer ${ }^{1 *}$

\begin{abstract}
${ }^{1}$ Department of Surgery, Leiden University Medical Center, Leiden, Netherlands, ${ }^{2}$ Curadel, Natick, MA, United States, ${ }^{3}$ Central Laboratory Animal Facility, Leiden University Medical Center, Leiden, Netherlands, ${ }^{4}$ Anatomy and Physiology Division, Faculty of Veterinary Medicine, Utrecht University, Utrecht, Netherlands, ${ }^{5}$ Department of Biomedical Sciences, Chonnam National University Medical School, Gwanju, South Korea, ${ }^{6}$ Department of Chemistry, Center for Diagnostics and Therapeutics, Georgia State University, Atlanta, GA, United States, ${ }^{7}$ Gordon Center for Medical Imaging, Department of Radiology, Massachusetts General Hospital and Harvard Medical School, Boston, MA, United States, ${ }^{8}$ Department of
\end{abstract} \\ Clinical Pharmacy and Toxicology, Leiden University Medical Center, Leiden, Netherlands
}

Assessing lymph node (LN) status during tumor resection is fundamental for the staging of colorectal cancer. Current guidelines require a minimum of $12 \mathrm{LNs}$ to be harvested during resection and ultra-staging regional lymph nodes by sentinel lymph node (SLN) assessment is being extensively investigated. The current study presents novel near-infrared (NIR) fluorescent dyes for simultaneous pan lymph node (PanLN; regional) and SLN mapping. PanLN-Forte was intravenously injected in mice and assessed for accumulation in regional LNs. SLN800 was injected intradermally in mice, after which the collection and retention of fluorescence in SLNs were measured using indocyanine green (ICG) and its precursor, SLN700, as references. LNs in the cervical, inguinal, jejunal, iliac, and thoracic basins could clearly be distinguished after a low dose intravenous injection of PanLN-Forte. Background fluorescence was significantly lower compared to the parent compound ZW800-3A ( $p<$ 0.001). SLN700 and SLN800 specifically targeted SLNs with fluorescence being retained over 40-fold longer than the current clinically used agent ICG. Using SLN700 and SLN800, absolute fluorescence in SLN was at least 10 times higher than ICG in second-tier nodes, even at 1 hour post-injection. Histologically, the fluorescent signal localized in the LN medulla (PanLN-Forte) or sinus entry (SLN700/SLN800). PanLN-Forte and SLN800 appear to be optimal for real-time NIR fluorescence imaging of regional and SLNs, respectively.

Keywords: image-guided surgery, fluorescence, cancer staging, ZW800, indocyanine green 


\section{INTRODUCTION}

Adequate yield and correct assessment of lymph node (LN) involvement in colorectal cancer (CRC) is of fundamental importance for determining the prognosis of cancer patients and guiding treatment decisions (1). Therefore, current guidelines of the National Comprehensive Cancer Network and European Society for Medical Oncology endorse a minimal harvest of $12 \mathrm{LNs}$ as quality indicator for CRC resections $(1,2)$. Obtaining an acceptable LN yield remains an important issue, especially in left-sided and smaller tumors, older patients and after neoadjuvant treatment where the minimal harvest is reached less often (3). In addition to gross lymph node examination, ultra-staging regional nodes by a sentinel lymph node (SLN) procedure is being investigated for refining staging and subsequent adjuvant therapy assignment (4). However, the sensitivity, ranging from 0.33 to 1.00 , remains an issue $(4,5)$. Both regional LN (all lymph nodes; referred to as PanLN henceforth) harvesting and SLN procedures could benefit from improved detection techniques.

Ionizing as well as non-ionizing agents based on near-infrared (NIR) light have been used to identified (S)LNs. The NIR I and II windows are advantageous due to the favorable penetration depth (up to 5-8 $\mathrm{mm}$ ), low tissue absorption and scattering, and minimal tissue autofluorescence (6). Consequently, the use of indocyanine green (ICG, a $800 \mathrm{~nm}$ NIR I fluorescent dye) for SLN-detection has been studied extensively in patients with malignancies of breast, skin, vulva, bladder, prostate, cervix, endometrium, ovarium, esophagus, stomach and colon (7-11). Unfortunately, ICG passes rapidly through the SLN towards deeper LNs, leading to unnecessary removal of these nodes (12). Another approach utilizes quantum dots to target SLNs as they have a high quantum yield (QY) and narrow bandwidth, permitting simultaneous visualization of up to five separate SLN dyes (13). However, their clinical translation is currently hampered due to their semiconductor-containing cores (13-16). In contrast to SLN tracers, research into panLN tracers is sparse. One approach using Cy5.5 polymers suffered from a high non-specific background signal after intravenous injection (17). By engineering novel small molecule-based polymethine cyanine fluorophores we recently visualized SLN as well as PanLNs at two distinct fluorescence emissions: $700 \mathrm{~nm}$ for the SLN agent and $800 \mathrm{~nm}$ for the PanLN agent, in small and large animal models (18). These dyes have meanwhile been refined with respect to their optical properties and pharmacokinetics.

The objective of the current study was to evaluate derivatized SLN and PanLN fluorophores for the detection of regional and sentinel LNs by in vivo NIR fluorescence imaging in mice. PanLN and SLN targeting efficiencies were measured in realtime using mice models, and post-mortem histological analyses were used to verify lymphatic uptake.

\section{MATERIALS AND METHODS}

\section{Syntheses of Contrast Agents}

Polymethine contrast agents generally have similar synthetic pathways that include a condensation reaction to combine two side groups with a central core. These side groups are usually functionalized indoles, whereas the central core contains the unsaturated hydrocarbon chain. In the case of the PanLN agents, the central core of the first NIR intermediate contains a halogen group that is used in a substitution reaction to add additional chemical groups to the molecule.

The reference compound ZW800-3A (19) and the novel compound PanLN-Forte (Figure 1A) syntheses start with the alkylation of commercially available 2,3,3-trimethyl-3H-indole with the salt (3-bromopropyl)trimethylammonium bromide. This indole is then used in a 2:1 ratio during a condensation reaction to produce the first NIR intermediate. The halogenated NIR intermediate is then used in an SN1 reaction with SOPP to form ZW800-3A, or used in a Suzuki-Miyaura coupling to form PanLN-Forte.

The SLN contrast agent's indoles are prepared with the alkylation of the commercially available 1,1,2-trimethylbenz[e] indole with iodoethane. The functionalized indole is then reacted with either a 3-carbon or 5-carbon central core to produce the reference compound SLN700 (18) and the novel compound SLN800 (Figure 1B), respectively.

All polymethine contrast agents were synthesized from chemicals and solvents that were of ACS grade or greater. Starting materials were purchased from Sigma-Aldrich (Saint Louis, MO) or Fisher Scientific Inc. (Pittsburgh, PA) and used without purification.

Final products were either purified by a series of solvent washes or by solid phase extraction using PoraPak Rxn sorbents (Waters, Milford, MA). Chemical identification and analysis of the contrast agents were completed on an Acquity UPLC-MS (Waters, Milford, MA).
A

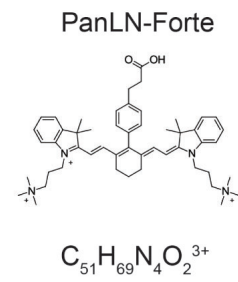

MW (Da): 770.115

abs, max: $756 \mathrm{~nm}$

emi, max: $774 \mathrm{~nm}$ QY: $15.4 \%$

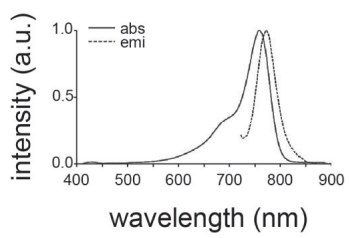

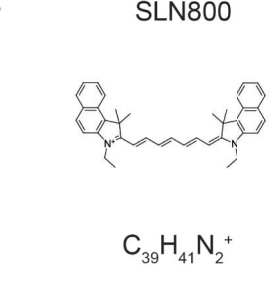

MW (Da): 537.753

abs, max: $802 \mathrm{~nm}$ emi, $\max : 823 \mathrm{~nm}$ QY: $15.0 \%$

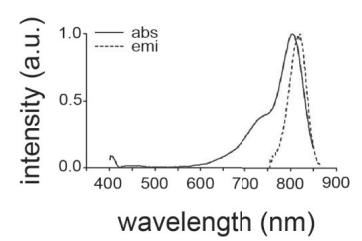

FIGURE 1 | Chemical structure and optical properties of $800 \mathrm{~nm}$ lymph node fluorophores in HEPES-buffered serum, pH 7.4. (A) PanLN-Forte and (B) SLN800. abs, absorption; a.u., arbitrary units; Da, Dalton; emi, emission; max, maximum; MW, molecular weight; nm, nanometer; QY, quantum yield. 


\section{Optical Property Analysis}

Optical properties were measured in fetal bovine serum (FBS) supplemented with $100 \mathrm{mM}$ HEPES, pH 7.4 or phosphatebuffered saline (PBS), pH 7.4. Quantum yields (QY) were determined in dimethyl sulfoxide with the comparative method using ICG (QY 13\%) as calibration standard. Optical measurements were collected on a Cary 50 Bio UV-Visible spectrophotometer, Cary Eclipse fluorescence spectrometer, respectively (Varian/Agilent, Mattapoisett, MA).

\section{Animal Models}

Animal experiments were approved by the Dutch Central Commission for Animal experimentation (Centrale Commissie voor Dierproeven). Mouse experiments were performed at the Central Animal Facility of the Leiden University Medical Center (LUMC). All animals were SPF as commended by the FELASA recommendation (20). Six-month-old female NMRI-mice (Charles River laboratories, l'Arbresle, France) were used for all experiments except for studies evaluating fluorescence retention in the SLN, where 6-10 week-old Foxn $1<$ nu $>$ mice (Charles River laboratories, l'Arbresle, France) were used. Each test condition consisted of three to five mice.

\section{NIR In Vivo Imaging Systems}

The Pearl Trilogy Small Animal Imaging System (LI-COR Biosciences, NE, USA) allows white-light and near-infrared imaging at 700 and $800 \mathrm{~nm}$ in a black-box setting, minimizing the interference from ambient light. It is used as a gold standard for quantification and comparison of in vivo fluorescent signals. To demonstrate the potential of the dyes for clinical translation the mini-FLARE Imaging Systems Kit, FLARE Model R1 (both Curadel, Natick, MA, USA) and Artemis (Quest Medical Imaging, Netherlands) near-infrared camera systems were used. Each of these systems is equipped with $700 \mathrm{~nm}$ and $800 \mathrm{~nm}$ channels next to visual color, and is suitable for intraoperative use. Using mini-FLARE, excitation wavelengths for $700 \mathrm{~nm}$ and 800 $\mathrm{nm}$ NIR fluorophores were $665 \pm 1 \mathrm{~nm}$ and $760 \pm 1 \mathrm{~nm}$, respectively, with typical fluence rates of $1-10 \mathrm{~mW} / \mathrm{cm} 2$.

\section{In Vivo LN Imaging}

PanLN-Forte and ZW800-3A (Curadel, Natick, MA, USA) were administered intravenously at the doses indicated in the tail vein of NMRI-mice. Four hours after injection the sub-iliac, proper axillary, accessory axillary, mandibular, accessory mandibular, superficial parotid, jejunal, medial iliac, external iliac, tracheobronchial and caudal mediastinal LNs were identified as described by Van de Broeck et al., followed by imaging and resection (21). Previous work demonstrated that $4 \mathrm{hrs}$ postinjection is the optimal imaging moment for PanLNs and $5 \mathrm{nmol}$ is the optimal dose for ZW800-3A, which served as reference (18). Accordingly, concentrations of 1, 5, and $10 \mathrm{nmol}$ PanLNForte in $100 \mu \mathrm{l}$ phosphate buffered saline (PBS) were tested. Biodistribution of the PanLN agents was determined at 5 minutes $(\mathrm{min})$ and $4 \mathrm{hrs}$ post-injection.

Ten microliters of 30, 125, and $500 \mu \mathrm{M}$ SLN700 and SLN800 dissolved in 50\% ethanol:PBS were administered intradermally in the footpad of NMRI-mice. SLNs were dissected, imaged, resected, and confirmed by histology (described below). Fluorescence retention in the SLN was assessed by intradermal injection of $10 \mu \mathrm{l}$ dye at the left side of the base of the tail in $\mathrm{Nu} /$ $\mathrm{Nu}$ mice. Lymph flow, first to the left sub-iliac LN (first tier node, SLN) and subsequently to the left axillary LNs (second tier node, distal LNs) was followed for a maximum of $15 \mathrm{~min}$. Imaging was repeated at $30 \mathrm{~min}$ and $60 \mathrm{~min}$, respectively, and after $24 \mathrm{hrs}$. All mice were kept under isoflurane anesthesia during injection and imaging. Based on previous studies, $500 \mu \mathrm{M}$ ICG was used as a control (22).

\section{Histological Analysis}

Resected tissue specimens were fixed overnight in $4 \%$ formalin, embedded in paraffin, sectioned $(5 \mu \mathrm{m})$, and scanned with the Odyssey Clx Infrared Imaging System for NIR-fluorescence (LICOR Biosciences, NE, USA). Sections were subsequently stained with hematoxylin-eosin, digitalized with the Pannoramic Digital Slide Scanner, and viewed with CaseViewer 2.3 (both 3D Histech, Hungary). A European certified veterinary pathologist (ECVP) confirmed the presence of lymphoid tissue. Merged images were generated for ultrastructural evaluation of the fluorescent location.

\section{Quantification and Statistical Analysis}

Pearl and Odyssey Clx images were analyzed with Image Studio Ver5.2 (LI-COR Biosciences, NE, USA). FLARE images were analyzed using FLARE-software (Curadel, Natick, MA, USA). Artemis images were captured with Spectrum Capture Suite 1.4.3 (Quest Medical Imaging, Netherlands) and analyzed with Fiji Image-J (23, 24). Signal-to-background ratios (SBRs) were measured by drawing a region of interest (ROI) around the LN of interest and a second ROI of similar size on the surrounding fat or muscle when no fat was present, and subsequent division of both mean fluorescent intensities (MFI). Results are reported as mean with standard deviation (SD). Means were compared using t-tests with IBM SPSS Statistics 23 (IBM Nederland BV, The Netherlands). Only p-values equal to or below 0.05 indicated significance.

\section{RESULTS}

\section{Optical and Chemical Properties}

PanLN-Forte has strong optical properties in serum with a maximum absorbance wavelength of $756 \mathrm{~nm}$ and emission wavelength of $774 \mathrm{~nm}$ (Figure 1A). The molar extension coefficient at $756 \mathrm{~nm}$ is $158,300 \mathrm{M}^{-1} \cdot \mathrm{cm}^{-1}$ and the QY is 15.4\%. SLN800 has a similar molar extinction coefficient at $156,300 \mathrm{M}^{-1} \cdot \mathrm{cm}^{-1}$, but with higher peak absorbance/emission wavelengths, and a QY of $15.0 \%$. The maximum absorbance and emission wavelengths for SLN800 are 802 and $823 \mathrm{~nm}$, respectively. SLN700 has a molar extinction coefficient of $157,400 \mathrm{M}^{-1} \cdot \mathrm{cm}^{-1}$, a maximum absorbance at $692 \mathrm{~nm}$, and a maximum emission wavelength of $714 \mathrm{~nm}$. Mass Spectrometry show $\mathrm{M}+3$ for PanLN-Forte and $\mathrm{M}+$ for SLN800 (Supplementary Figure 1). 


\section{PanLN Imaging}

PanLN-Forte specifically accumulated in clinically important superficial, abdominal and thoracic LNs, and the MFI increased with dose (Figures 2A, B, Supplementary Video 1). With all three dose groups and NIR imaging systems SBRs were $>2.0$ except for the $1 \mathrm{nmol}$ dose group (Supplementary Table 1). While the MFI values of the LNs after PanLN-Forte injection were similar to those of the parent compound ZW800-3A ( $\mathrm{p}=$ 0.753 , Figure 2B), the non-specific signal in the liver was significantly reduced $(\mathrm{p}<0.001$, Figure $2 \mathrm{C}$ ). Biodistribution analysis revealed that PanLN-Forte was excreted into the bile within $5 \mathrm{~min}$ after injection. Non-specific fluorescence had cleared sufficiently after 4 hrs to allow jejunal LN visualization as the MFI of the jejunal LNs was $>2$ higher than all organs except for the liver (Figure 2D).

\section{SLN Imaging}

SLNs could be visualized at a lower dose of SLN800 than the clinically used ICG dose (125 $\mu \mathrm{M}$ vs $500 \mu \mathrm{M}$, Figure 3A). SBRs for $500 \mu \mathrm{M}$ and $125 \mu \mathrm{M}$ SLN800 were similar and differed significantly from $31 \mu \mathrm{M}$ SLN800 $(125 \mu \mathrm{M}$ vs. $31 \mu \mathrm{M}: \mathrm{p}=$ 0.008 , Figure 3B). The optimal dose of SLN800 was set at 125 $\mu \mathrm{M}$, as it was the lowest dose that still resulted in the requisite SBR of $>2$ with all three fluorescent imaging systems. For SLN700 the optimal dose was set at $500 \mu \mathrm{M}$, as this gave the highest SBRs $(500 \mu \mathrm{M}$ vs. $125 \mu \mathrm{M}: \mathrm{p}=0.001 ; 500 \mu \mathrm{M}$ vs. $31 \mu \mathrm{M}$ : $\mathrm{p}=0.008$, Figure $3 \mathrm{~B}$ ). While ICG rapidly passed to distal nodes (non-SLN), SLNs retained SLN700 and SLN800 over 40 times longer ( $14 \pm 3$ seconds vs. $17 \pm 9 \mathrm{~min}$ vs. $10 \pm 7 \mathrm{~min}$, respectively, Figure 3A). Using SLN700 and SLN800, absolute fluorescence in the SLN was at least ten times higher than that in second-tier nodes, even at $1 \mathrm{hr}$ post-injection (Figure 3C). This allowed for discrimination of SLNs from second-tier nodes using MFI long after the fluorescence has passed the SLN with SLN700 and SLN800.

\section{Ultrastructural Fluorescence of LN Dyes}

Intravenous injection of PanLN-Forte identified 94 LNs in 13 mice. All LNs in the $5 \mathrm{nmol}$ dose group were resected for histology and contained lymphoid tissue. Histologically, the fluorescence localized towards the medulla of the LN (Figure 4A)

Twenty-nine of 31 SLNs identified with SLN800 were histologically confirmed as lymph nodes. Although visually confirmed during resection, 2 SLNs could not be histologically verified due to loss or damage of tissue during surgical resection and subsequent histological preparation. All 19 SLNs found with SLN700 were histologically confirmed. Fluorescence accumulating in the subcapsular sinus confirmed lymphatic sinus entry of the dye (Figure $\mathbf{4 B}$ ).
A
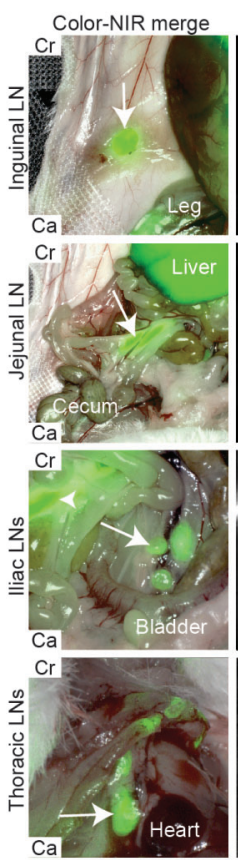

NIR $800 \mathrm{~nm}$
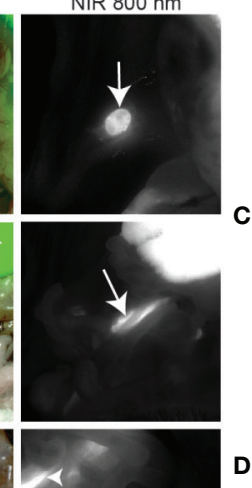

B
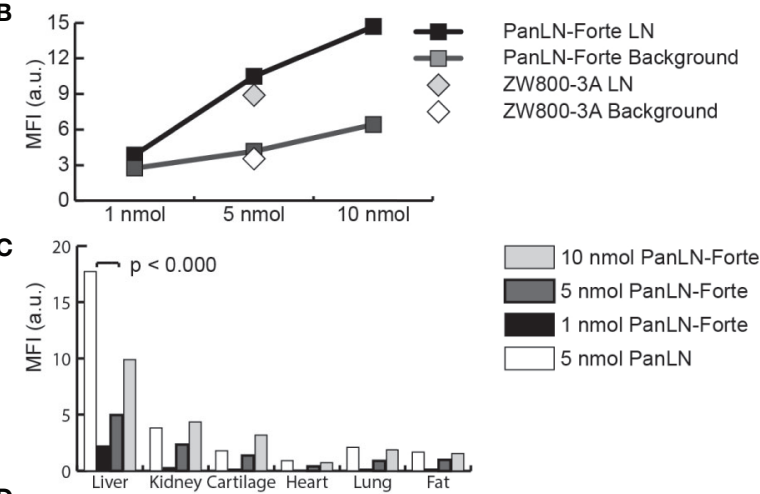

D

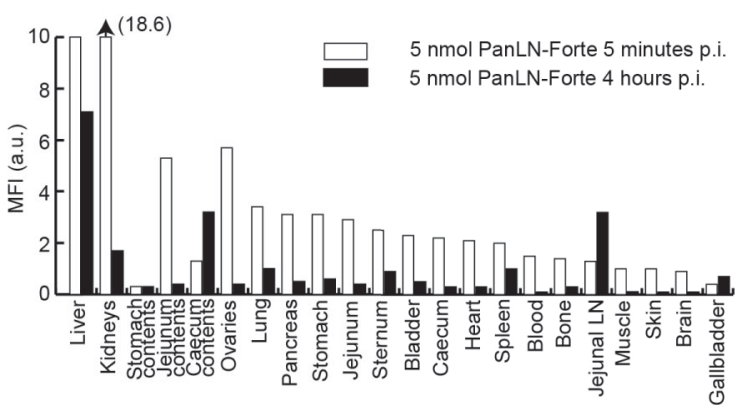

FIGURE 2 | Regional lymph node imaging with PanLN-Forte at > $780 \mathrm{~nm}$ emission. (A) Important LNs located in superficial fat (inguinal LN), the abdomen (jejunal and iliac LNs), and thorax could be distinguished from the background $4 \mathrm{hrs}$ after injection of PanLN-Forte. Arrows indicate the respective LN while the arrowhead points to the jejunal LNs. Images taken with the FLARE Model R1. (B) MFI of signal and background for ZW800-3a and PanLN-Forte. (C) Dose-dependent uptake ZW800-3a vs. PanLN-Forte. (D) Biodistribution at 5 min and 4 hrs after injection of PanLN. a.u., arbitrary unit; Ca, caudal; Cr, cranial; LN, lymph node; MFI, mean fluorescent intensity; NIR, near-infrared; nm, nanometer; nmol, nanomole; p.i., post injection. 


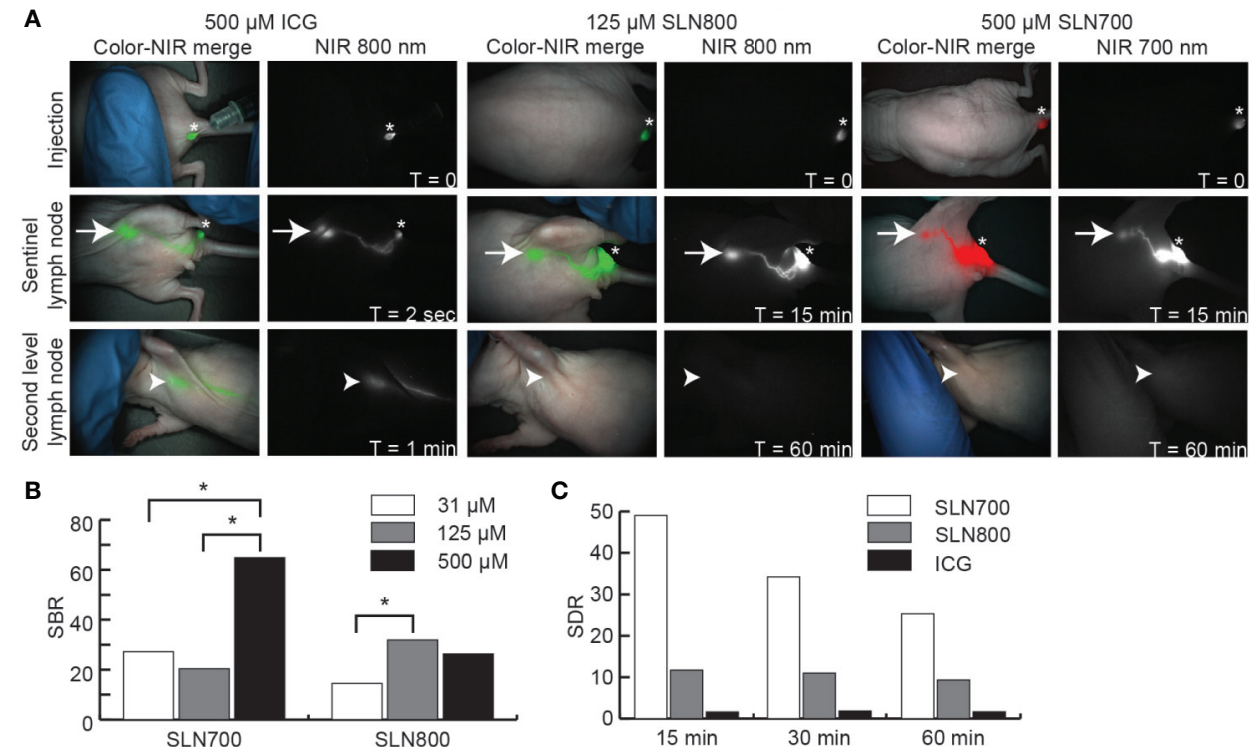

FIGURE 3 | Near-infrared sentinel lymph node imaging. (A) NIR fluorescent images illustrating over time (0-60 min) the fluorescence at the injection site, SLN and second tier node (distal node) after intra-dermal ICG, SLN800, and SLN700 injection. Asterisks indicate injection sites, arrows the SLN and arrowheads the location of second tier nodes. Images taken with the Artemis excited with a 785 laser for ICG and SLN800 and 680 nm laser for SLN700. (B) SBRs of various doses SLN700 and SLN800. (C) SLN-to-distal node ratio (SDR) of SLN700, SLN800, and ICG over time (0-60 min). ICG, indocyanine green; SLN, sentinel lymph node; $\mu$ M, micromolar; min, minutes; NIR, near-infrared; nm, nanometer; SBR, signal-to-background ratio; SDR, SLN-to-distal node ratio; sec, seconds; T, times.

A

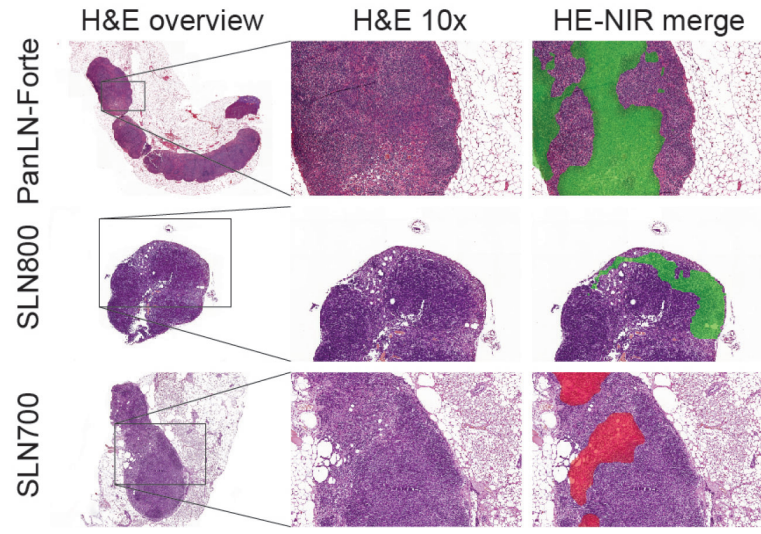

FIGURE 4 | Ultrastructural analysis of lymph node fluorescence. (A) PanLNForte. $800 \mathrm{~nm}$ fluorescence (green pseudo-color) was seen throughout the medulla. (B) SLN800 and SLN700. $800 \mathrm{~nm}$ fluorescence (green pseudocolor) and $700 \mathrm{~nm}$ fluorescence (red pseudo-color) localized to the subcapsular sinus after intra-dermal injection. An overview H\&E staining and 10x magnification are shown, after which the 10x H\&E was merged with the fluorescence images. H\&E, hematoxylin \& eosin; NIR, near-infrared.

\section{DISCUSSION}

In $\mathrm{CRC}$, resecting a minimal of $12 \mathrm{LNs}$ has been related to prognosis and survival outcomes and therefore been incorporated in various international guidelines (1-3). Consequently, the proportion of surgeries in which 12 or more LNs have been resected has risen from $13 \%-35 \%$ at the turn of the century to $59 \%-74 \%(25-28)$. While the majority of LN metastasis in CRC are found in nodes smaller than five millimeter, the current techniques of visualization and palpation bias's towards identifying larger LNs (29). Current navigation techniques do not identify PanLNs. Furthermore, SLN-biopsy investigations are hampered by the currently available SLN-agents as they are neither real-time, retained in the SLN, aesthetically pleasing due to tattooing, nor conserving of the surgical field.

The current in vivo study describes improved PanLN and SLN imaging agents ready for clinical translation. After intravenous injection, PanLN-Forte exhibited reduced background signal in key organs such as the liver, unlike the previously described Cy5.5 polymers and ZW800-3A, while maintaining PanLN homing ability $(17,18)$. Despite finding uptake of PanLN-Forte in the liver and, to a lesser extent, in cartilage, these structures can be shielded during a surgical procedure, and are generally far away from the mesenteric LNs that play a relevant role in CRC LN metastasis (30). The optimal dose of $5 \mathrm{nmol}$ translates to a human equivalent dose of $0.015 \mathrm{mg} / \mathrm{kg}$, which is a low dose and similar to that of other recently clinically tested fluorophores for anatomic enhancement $(31,32)$. Histologically, PanLN-Forte accumulated in the medulla of LNs suggesting direct uptake from the systemic circulation. Although the exact mechanism of uptake is not currently known, phagocytic cells, such as alveolar macrophages and dendritic cells, preferentially engulf cationic structures (33).

SLN800 identified SLNs in real-time and with a substantially lower dose than ICG $(125 \mu \mathrm{M}$ vs. $500 \mu \mathrm{M})$. Partially, this can be attributed to the higher QY of SLN800 than ICG (15\% vs. 9\%) (34). Furthermore, SLN800 and SLN700 were retained in the SLN over 40 
times longer than ICG. The improved performance of the SLN dyes versus ICG is not understood completely. Typically, an ideal agent for SLN targeting is large ( $\geq 10-50 \mathrm{~nm}$ hydrodynamic diameter) and has high charge, high hydrophobicity, or both $(35,36)$. Hence, the rapid clearance of ICG out of the lymphatics can be attributed to its small hydrodynamic diameter of $1.2 \mathrm{~nm}$ and amphiphilic structure. Previously, ICG has been mixed with human serum albumin (HSA) to increase its size to $7.3 \mathrm{~nm}$, but in clinical trials, ICG:HSA did not result in improved SLN detection over ICG alone (37). The chemical structures of SLN700 and SLN800 offer clues as to why they have such high retention compared to ICG. SLN800 and SLN700 are both much more hydrophobic than ICG and have a cationic charge from the indole nitrogen. This combination of charge and hydrophobicity either increases effective hydrodynamic diameter by binding to proteins in lymph, or is a trigger for uptake by phagocytic lymphatic cells at the sinus entry point. Either way, the net effect is higher retention in the SLN.

As the NIR fluorescence imaging field evolves, and as AIdirected robotic surgery commences, dual-wavelength (i.e., multiplexed) navigation will become increasingly important. With the introduction of two-channel NIR-camera systems operating with excitations at $700 \mathrm{~nm}$ as well as $800 \mathrm{~nm}$, surgeons and/or robots can focus on two targets at the same time. The combined optical properties of PanLN-Forte and SLN700 permit simultaneous visualization of both the SLN and all other nodes in a tumor basin. Furthermore, in colorectal cancer the combination of PanLN-Forte with SGM-101, a $700 \mathrm{~nm}$ CEA-targeting tumor-specific agent currently in phase III trials (NCT03659448), would facilitate the identification of both the tumor and the LNs (38). This is especially relevant in rectal cancer patients treated with neoadjuvant (chemo) radiotherapy where extensive fibrosis and scar tissue make tumor and LN identification difficult (39). SLN700 and SLN800 also permit concurrent SLN and tumor imaging with the whole arsenal of tracers in various study protocols, and future development may permit the use of dual-wavelength NIR fluorescence to both find the SLN and identify micro-metastases in real-time (40). Finally, the potential of SLN700, SLN800 and PanLN-Forte is not only limited to colorectal cancer but also applicable to other tumor types like non-small cell lung cancer where a minimum of 10 LNs need to be resected and vulvar, headand-neck and esophageal cancer where SLN-biopsies are being studied extensively $(7,41)$.

A possible limitation of the current study is that animal experiments were not performed in tumor-bearing mice, which could have altered lymph drainage. However, previous studies suggest no difference in tumor-bearing and non-tumor-bearing animals with respect to SLN identification (42). Another limitation might arise during clinical translation, where high BMI, previous neoadjuvant therapy, or previous abdominal procedures could hamper LN visualization. In fact, visual enhancement methods are most needed in these clinical settings (43).

The novel $800 \mathrm{~nm}$ contrast agents PanLN-Forte and SLN800 appear to be optimal for real-time NIR fluorescence imaging of regional and sentinel lymph nodes, respectively, may be used in conjugation with $700 \mathrm{~nm}$ contrast agents for multi-wavelength surgical guidance, and rapid clinical translation is expected.

\section{DATA AVAILABILITY STATEMENT}

The raw data supporting the conclusions of this article will be made available by the authors upon reasonable request.

\section{ETHICS STATEMENT}

The Dutch Central Commission for Animal Experimentation approved all animal experiments (AVD1160020172925). Experiments were performed in accordance with the code of practice "Dierproeven In Het Kankeronderzoek".

\section{AUTHOR CONTRIBUTIONS}

The manuscript has been seen and approved by all authors. VB, $\mathrm{MD}$, and SB executed the preclinical studies. DS trained the preclinical researchers and analyzed the histological data. $\mathrm{MB}$, $\mathrm{HH}, \mathrm{MH}$, and $\mathrm{HC}$ developed the dyes. MB, AT, TM, and AV were responsible for the production of the dyes and analysis of chemical data. CS, PK, JF, and AV coordinated and supervised the experiments. VB wrote the manuscript with assistance from all the contributing authors. All authors contributed to the article and approved the submitted version.

\section{FUNDING}

Research reported in this study was supported in part by the National Cancer Institute (NCI) of the National Institutes of Health under award number R01-CA-207500. The content is solely the responsibility of the authors and does not necessarily represent the official views of the National Institutes of Health. CS was in part funded by the European Commission under two Marie Skłodowska-Curie Action awards: H2020-MSCA-RISE2019 (Project number: 872860 - PRISAR2) and H2020-MSCAITN-2019 (Project number: 857894 - CAST).

\section{ACKNOWLEDGMENTS}

The authors would like to acknowledge R.L.P. Vlierberghe and N.G. Dekker-Ensink for their excellent technical support.

\section{SUPPLEMENTARY MATERIAL}

The Supplementary Material for this article can be found online at: https://www.frontiersin.org/articles/10.3389/fonc.2020. 586112/full\#supplementary-material

SUPPLEMENTARY VIDEO 1 | NIR identification and resection of LNs after injection of $5 \mathrm{nmol}$ PanLN-Forte. Fluorescence imaging was done using the Artemis (Quest, Netherlands). LNs were confirmed by pathology. 


\section{REFERENCES}

1. Schmoll HJ, Van Cutsem E, Stein A, Valentini V, Glimelius B, Haustermans $\mathrm{K}$, et al. ESMO Consensus Guidelines for management of patients with colon and rectal cancer. a personalized approach to clinical decision making. Ann Oncol (2012) 23(10):2479-516. doi: 10.1093/annonc/mds236

2. Engstrom PF, Arnoletti JP, Benson AB,3, Chen YJ, Choti MA, Cooper HS, et al. NCCN Clinical Practice Guidelines in Oncology: colon cancer. J Natl Compr Cancer Netw (2009) 7(8):778-831. doi: 10.6004/jnccn.2009.0056

3. Orsenigo E, Gasparini G, Carlucci M. Clinicopathological Factors Influencing Lymph Node Yield in Colorectal Cancer: A Retrospective Study. Gastroenterol Res Pract (2019) 2019:5197914. doi: 10.1155/2019/5197914

4. Scabini S. Sentinel node biopsy in colorectal cancer: Must we believe it? World J Gastrointest Surg (2010) 2(1):6-8. doi: 10.4240/wjgs.v2.i1.6

5. van der Zaag ES, Bouma WH, Tanis PJ, Ubbink DT, Bemelman WA, Buskens CJ. Systematic review of sentinel lymph node mapping procedure in colorectal cancer. Ann Surg Oncol (2012) 19(11):3449-59. doi: 10.1245/ s10434-012-2417-0

6. Gioux S, Choi HS, Frangioni JV. Image-guided surgery using invisible nearinfrared light: fundamentals of clinical translation. Mol Imaging (2010) 9 (5):237-55. doi: 10.2310/7290.2010.00034

7. van Manen L, Handgraaf HJM, Diana M, Dijkstra J, Ishizawa T, Vahrmeijer $\mathrm{AL}$, et al. A practical guide for the use of indocyanine green and methylene blue in fluorescence-guided abdominal surgery. J Surg Oncol (2018) 118 (2):283-300. doi: 10.1002/jso.25105

8. van der Vorst JR, Hutteman M, Gaarenstroom KN, Peters AA, Mieog JS, Schaafsma BE, et al. Optimization of near-infrared fluorescent sentinel lymph node mapping in cervical cancer patients. Int J Gynecol Cancer (2011) 21 (8):1472-8. doi: 10.1097/IGC.0b013e31822b451d

9. Schaafsma BE, Mieog JS, Hutteman M, van der Vorst JR, Kuppen PJ, Lowik $\mathrm{CW}$, et al. The clinical use of indocyanine green as a near-infrared fluorescent contrast agent for image-guided oncologic surgery. J Surg Oncol (2011) 104 (3):323-32. doi: 10.1002/jso.21943

10. Mieog JS, Troyan SL, Hutteman M, Donohoe KJ, van der Vorst JR, Stockdale A, et al. Toward optimization of imaging system and lymphatic tracer for near-infrared fluorescent sentinel lymph node mapping in breast cancer. Ann Surg Oncol (2011) 18(9):2483-91. doi: 10.1245/s10434-011-1566-X

11. Hutteman M, Choi HS, Mieog JS, van der Vorst JR, Ashitate Y, Kuppen PJ, et al. Clinical translation of ex vivo sentinel lymph node mapping for colorectal cancer using invisible near-infrared fluorescence light. Ann Surg Oncol (2011) 18(4):1006-14. doi: 10.1245/s10434-010-1426-0

12. Ahmed M, Purushotham AD, Douek M. Novel techniques for sentinel lymph node biopsy in breast cancer: a systematic review. Lancet Oncol (2014) 15(8): e351-62. doi: 10.1016/S1470-2045(13)70590-4

13. Kobayashi H, Hama Y, Koyama Y, Barrett T, Regino CA, Urano Y, et al. Simultaneous multicolor imaging of five different lymphatic basins using quantum dots. Nano Lett (2007) 7(6):1711-6. doi: 10.1021/nl0707003

14. Lim YT, Kim S, Nakayama A, Stott NE, Bawendi MG, Frangioni JV. Selection of quantum dot wavelengths for biomedical assays and imaging. Mol Imaging (2003) 2(1):50-64. doi: 10.1162/153535003765276282

15. Frangioni JV, Kim SW, Ohnishi S, Kim S, Bawendi MG. Sentinel lymph node mapping with type-II quantum dots. Methods Mol Biol (Clifton NJ) (2007) 374:147-59. doi: 10.1385/1-59745-369-2:147

16. Hauck TS, Anderson RE, Fischer HC, Newbigging S, Chan WC. In vivo quantum-dot toxicity assessment. Small (Weinheim an der Bergstrasse Germany) (2010) 6(1):138-44. doi: 10.1002/smll.200900626

17. Josephson L, Mahmood U, Wunderbaldinger P, Tang Y, Weissleder R. Pan and sentinel lymph node visualization using a near-infrared fluorescent probe. Mol Imaging (2003) 2(1):18-23. doi: 10.1162/153535003765276255

18. Ashitate Y, Hyun H, Kim SH, Lee JH, Henary M, Frangioni JV, et al. Simultaneous mapping of pan and sentinel lymph nodes for real-time image-guided surgery. Theranostics (2014) 4(7):693-700. doi: 10.7150/thno.8721

19. Choi HS, Nasr K, Alyabyev S, Feith D, Lee JH, Kim SH, et al. Synthesis and in vivo fate of zwitterionic near-infrared fluorophores. Angewandte Chemie (International ed English) (2011) 50(28):6258-63. doi: 10.1002/ anie.201102459

20. Mahler Convenor M, Berard M, Feinstein R, Gallagher A, Illgen-Wilcke B, Pritchett-Corning $\mathrm{K}$, et al. FELASA recommendations for the health monitoring of mouse, rat, hamster, guinea pig and rabbit colonies in breeding and experimental units. Lab Anim (2014) 48(3):178-92. doi: $10.1177 / 0023677213516312$

21. Van den Broeck W, Derore A, Simoens P. Anatomy and nomenclature of murine lymph nodes: Descriptive study and nomenclatory standardization in BALB/cAnNCrl mice. J Immunol Methods (2006) 312(1-2):12-9. doi: 10.1016/ j.jim.2006.01.022

22. Schaafsma BE, van der Vorst JR, Gaarenstroom KN, Peters AA, Verbeek FP, de Kroon CD, et al. Randomized comparison of near-infrared fluorescence lymphatic tracers for sentinel lymph node mapping of cervical cancer. Gynecol Oncol (2012) 127(1):126-30. doi: 10.1016/j.ygyno.2012.07.002

23. Schindelin J, Arganda-Carreras I, Frise E, Kaynig V, Longair M, Pietzsch T, et al. Fiji: an open-source platform for biological-image analysis. Nat Methods (2012) 9(7):676-82. doi: 10.1038/nmeth.2019

24. Rueden CT, Schindelin J, Hiner MC, DeZonia BE, Walter AE, Arena ET, et al. ImageJ2: ImageJ for the next generation of scientific image data. BMC Bioinformatics (2017) 18(1):529. doi: 10.1186/s12859-017-1934-Z

25. Parsons HM, Tuttle TM, Kuntz KM, Begun JW, McGovern PM, Virnig BA. Association between lymph node evaluation for colon cancer and node positivity over the past 20 years. JAMA (2011) 306(10):1089-97. doi: 10.1001/jama.2011.1285

26. van Erning FN, Crolla RM, Rutten HJ, Beerepoot LV, van Krieken JH, Lemmens VE. No change in lymph node positivity rate despite increased lymph node yield and improved survival in colon cancer. Eur J Cancer (Oxford Engl 1990) (2014) 50(18):3221-9. doi: 10.1016/j.ejca.2014.10.011

27. Bui L, Rempel E, Reeson D, Simunovic M. Lymph node counts, rates of positive lymph nodes, and patient survival for colon cancer surgery in Ontario, Canada: a population-based study. J Surg Oncol (2006) 93(6):439_ 45. doi: 10.1002 /jso.20499

28. Wright FC, Law CH, Last L, Khalifa M, Arnaout A, Naseer Z, et al. Lymph node retrieval and assessment in stage II colorectal cancer: a population-based study. Ann Surg Oncol (2003) 10(8):903-9. doi: 10.1245/ASO.2003.01.012

29. Rodriguez-Bigas MA, Maamoun S, Weber TK, Penetrante RB, Blumenson LE, Petrelli NJ. Clinical significance of colorectal cancer: metastases in lymph nodes $<5$ $\mathrm{mm}$ in size. Ann Surg Oncol (1996) 3(2):124-30. doi: 10.1007/BF02305790

30. Kim HJ, Choi GS. Clinical Implications of Lymph Node Metastasis in Colorectal Cancer: Current Status and Future Perspectives. Ann Coloproctol (2019) 35(3):109-17. doi: 10.3393/ac.2019.06.12

31. Nair AB, Jacob S. A simple practice guide for dose conversion between animals and human. J Basic Clin Pharm (2016) 7(2):27-31. doi: 10.4103/09760105.177703

32. de Valk KS, Handgraaf HJ, Deken MM, Sibinga Mulder BG, Valentijn AR, Terwisscha van Scheltinga AG, et al. A zwitterionic near-infrared fluorophore for real-time ureter identification during laparoscopic abdominopelvic surgery. Nat Commun (2019) 10(1):3118. doi: 10.1038/s41467-019-11014-1

33. Fromen CA, Rahhal TB, Robbins GR, Kai MP, Shen TW, Luft JC, et al. Nanoparticle surface charge impacts distribution, uptake and lymph node trafficking by pulmonary antigen-presenting cells. Nanomedicine (2016) 12 (3):677-87. doi: 10.1016/j.nano.2015.11.002

34. Ohnishi S, Lomnes SJ, Laurence RG, Gogbashian A, Mariani G, Frangioni JV. Organic alternatives to quantum dots for intraoperative near-infrared fluorescent sentinel lymph node mapping. Mol Imaging (2005) 4(3):172-81. doi: 10.1162/15353500200505127

35. Khullar O, Frangioni JV, Grinstaff M, Colson YL. Image-guided sentinel lymph node mapping and nanotechnology-based nodal treatment in lung cancer using invisible near-infrared fluorescent light. Semin Thorac Cardiovasc Surg (2009) 21(4):309-15. doi: 10.1053/j.semtcvs.2009.11.009

36. Ali Khan A, Mudassir J, Mohtar N, Darwis Y. Advanced drug delivery to the lymphatic system: lipid-based nanoformulations. Int J Nanomedicine (2013) 8:2733-44. doi: 10.2147/IJN.S41521

37. Hutteman M, Mieog JS, van der Vorst JR, Liefers GJ, Putter H, Lowik CW, et al. Randomized, double-blind comparison of indocyanine green with or without albumin premixing for near-infrared fluorescence imaging of sentinel lymph nodes in breast cancer patients. Breast Cancer Res Treat (2011) 127 (1):163-70. doi: 10.1007/s10549-011-1419-0

38. Boogerd LSF, Hoogstins CES, Schaap DP, Kusters M, Handgraaf HJM, van der Valk MJM, et al. Safety and effectiveness of SGM-101, a fluorescent antibody targeting carcinoembryonic antigen, for intraoperative detection of 
colorectal cancer: a dose-escalation pilot study. Lancet Gastroenterol Hepatol (2018) 3(3):181-91. doi: 10.1016/S2468-1253(17)30395-3

39. McDonald JR, Renehan AG, O’Dwyer ST, Haboubi NY. Lymph node harvest in colon and rectal cancer: Current considerations. World J Gastrointest Surg (2012) 4(1):9-19. doi: 10.4240/wjgs.v4.i1.9

40. Hernot S, van Manen L, Debie P, Mieog JSD, Vahrmeijer AL. Latest developments in molecular tracers for fluorescence image-guided cancer surgery. Lancet Oncol (2019) 20(7):e354-e67. doi: 10.1016/S1470-2045(19)30317-1

41. Samayoa AX, Pezzi TA, Pezzi CM, Greer Gay E, Asai M, Kulkarni N, et al. Rationale for a Minimum Number of Lymph Nodes Removed with NonSmall Cell Lung Cancer Resection: Correlating the Number of Nodes Removed with Survival in 98,970 Patients. Ann Surg Oncol (2016) 23(Suppl 5):1005-11. doi: 10.1245/s10434-016-5509-4

42. Kwon S, Sevick-Muraca EM. Functional lymphatic imaging in tumor-bearing mice. J Immunol Methods (2010) 360(1-2):167-72. doi: 10.1016/j.jim.2010. 06.016

43. Fujieda Y, Maeda H, Oba K, Okamoto K, Shiga M, Fujisawa K, et al. Factors influencing the number of retrieved lymph nodes after colorectal resection: a retrospective study from a single institute. Int J Clin Exp Pathol (2018) 11 (3):1694-700.

Conflict of Interest: Author JF is founder and CEO of Curadel, LLC. Author MB was employed by the company Curadel, LLC.

The remaining authors declare that the research was conducted in the absence of any commercial or financial relationships that could be construed as a potential conflict of interest.

Copyright (c) 2020 Baart, Deken, Bordo, Bhairosingh, Salvatori, Hyun, Henary, Choi, Sier, Kuppen, van Scheltinga, March, Valentijn, Frangioni and Vahrmeijer. This is an open-access article distributed under the terms of the Creative Commons Attribution License (CC BY). The use, distribution or reproduction in other forums is permitted, provided the original author(s) and the copyright owner(s) are credited and that the original publication in this journal is cited, in accordance with accepted academic practice. No use, distribution or reproduction is permitted which does not comply with these terms. 